\title{
Characterization and coating stability evaluation of nickel-titanium orthodontic esthetic wires: an in vivo study
}

\begin{abstract}
Nina ARGALJI(a)
Eduardo Moreira da SILVA(b)

Adriana CURY-SARAMAGO(a) Claudia Trindade MATTOS(a)
\end{abstract}

(a) Universidade Federal Fluminense, Department of Orthodontics, Niterói, RJ, Brazil.

(b) Universidade Federal Fluminense, School of Dentistry, Analytical Laboratory of Restorative Biomaterials - LABiom-R, Niterói, RJ, Brazil.
Declaration of Interest: The authors certify that they have no commercial or associative interest that represents a conflict of interest in connection with the manuscript.

\section{Corresponding Author:}

Claudia Trindade Mattos

claudiatrindademattos@gmail.com

Submitted: Jan 25, 2017

Accepted for publication: May 03, 2017

Last revision: June 06, 2017
Abstract: The objective of this study was to compare coating dimensions and surface characteristics of two different esthetic covered nickel-titanium orthodontic rectangular archwires, as-received from the manufacturer and after oral exposure. The study was designed for comparative purposes. Both archwires, as-received from the manufacturer, were observed using a stereomicroscope to measure coating thickness and inner metallic dimensions. The wires were also exposed to oral environment in 11 orthodontic active patients for 21 days. After removing the samples, stereomicroscopy images were captured, coating loss was measured and its percentage was calculated. Three segments of each wire (one as-received and two after oral exposure) were observed using scanning electron microscopy for a qualitative analysis of the labial surface of the wires. The Lilliefors test and independent $\mathrm{t}$-test were applied to verify normality of data and statistical differences between wires, respectively. The significance level adopted was 0.05 . The results showed that the differences between the wires while comparing inner height and thickness were statistically significant $(\mathrm{p}<0.0001)$. In average, the most recently launched wire presented a coating thickness twice that of the control wire, which was also a statistically significant difference. The coating loss percentage was also statistically different $(\mathrm{p}=0.0346)$ when the latest launched wire $(13.27 \%)$ was compared to the control (29.63\%). In conclusion, the coating of the most recent wire was thicker and more uniform, whereas the control had a thinner coating on the edges. After oral exposure, both tested wires presented coating loss, but the most recently launched wire exhibited better results.

Keywords: Orthodontic Wires; Orthodontics; Orthodontic Appliance Design

\section{Introduction}

The esthetic perception of patients has increased the demand for less noticeable orthodontic appliances, ${ }^{1}$ mainly in adults. ${ }^{2,3,4}$ The use of ceramic brackets partially solved this concern because they mimic tooth color. ${ }^{1,3,5,6}$ However, esthetic arches are still not used widely because their coating is not durable. ${ }^{5}$ In addition, the surface properties of these wires, which may influence treatment mechanics, may be altered during coating application. ${ }^{6}$ 
Authors have evaluated optical, biological and mechanical properties, such as sliding properties, $78,9,10$ coating stability, $2,8,11,12,13$ force transfer values, ${ }^{5,14}$ color stability,,$^{15}$ and plaque accumulation ${ }^{1}$ of esthetic archwires and observed that most of these characteristics are not yet ideal. ${ }^{16}$

Despite these disadvantages, esthetic wires are still commercialized and used in clinical practice. ${ }^{5}$ Manufacturers have been dedicated to continuous improvement aiming for properties that are sufficient or comparable to traditional wires. ${ }^{15,16}$

Insufficient data are available comparing properties of as-received coated wires ${ }^{10}$ and orally exposed wires, ${ }^{1,15}$ since most of the tests have been conducted in laboratories and do not simulate real clinical conditions. ${ }^{14}$

The objective of the present study was to characterize the dimensions, the surface features, and the coating stability of two types of nickel-titanium orthodontic archwires from the same brand in two conditions: as-received from the manufacturer and after oral exposure of 21 days.

\section{Methods}

Two types of rectangular nickel-titanium esthetic orthodontic wires from Forestadent (Pforzheim, Germany) were evaluated in this study: Titanol Cosmetic (0.018" x 0.024") and Biocosmetic $\left(0.018^{\prime \prime} \times 0.025^{\prime \prime}\right)$, which present Teflon and polymeric coating, respectively. ${ }^{4}$ All archwires were pre-contoured, the anterior curved region was discarded and only straight segments were used. Seven 10-mm segments produced from each type of archwire were used for coating thickness and dimension analyses $(n=5)$, surface characterization via scanning electron microscope-SEM, $(n=1)$ and 3D profilometry analysis $(n=1)$. Furthermore, eleven $20-\mathrm{mm}$ segments of each archwire $(n=11)$ were submitted to oral exposure.

\section{Coating thickness and dimensions characterization}

The specimens used for coating thickness and inner dimension analysis were embedded in a light-curing resin composite (Natural Flow,Nova DFL, Rio de Janeiro, Brazil), inside PVC cylinders (6-mm-high and 7-mm-diameter), with their transverse sections facing up. Then, the exposed transverse sections were mechanically wet polished using 600- and 1200-grit SiC papers (Aropol ED, Arotec, São Paulo, Brazil).

Images of the transverse sections were obtained with a stereomicroscope at 45X magnification (Olympus SZ61TR, Tokyo, Japan) and transferred to a computer. The coating thickness at three randomly spaced points in each coated surface and the inner height and width dimensions of each transverse section were measured using Image Pro Premier software (Media Cybernetics, Rockville, USA), with an accuracy of $0.0001 \mathrm{~mm}$ (Figure 1).
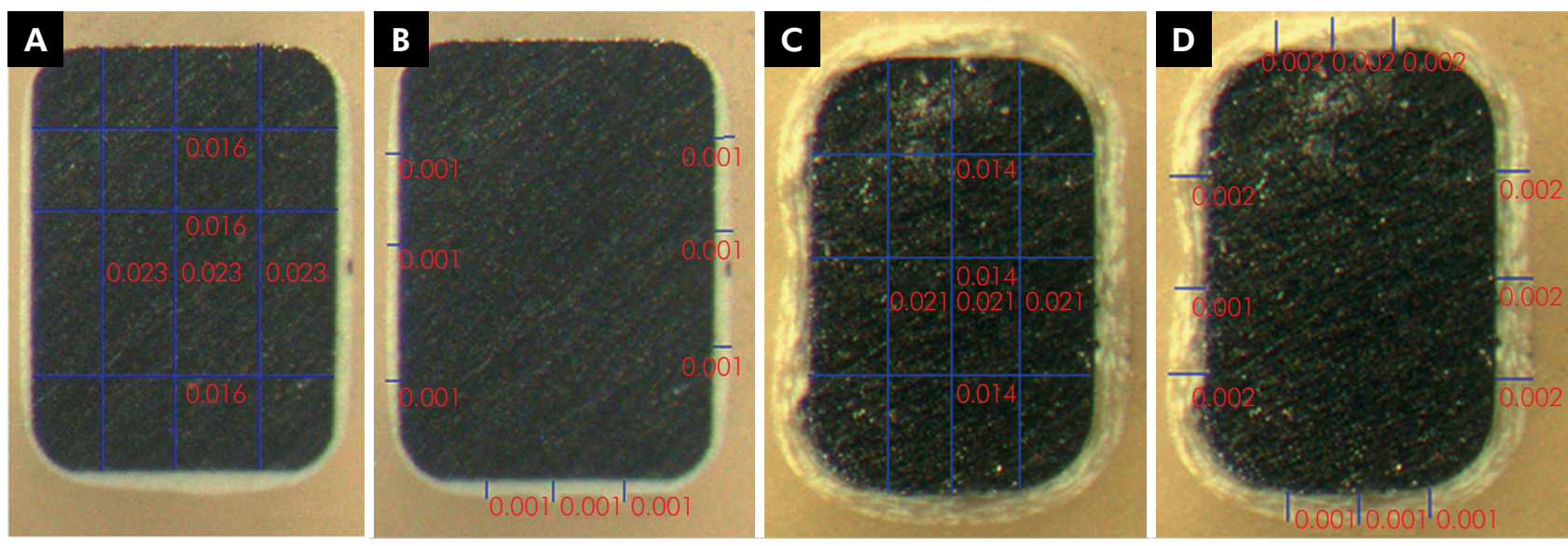

Figure 1. Stereomicroscope images of the transverse section illustrating the following measurements: A) Titanol Cosmetic inner dimensions; B) Titanol Cosmetic coating thickness; C) Biocosmetic inner dimensions; D) Biocosmetic coating thickness. Original magnification 45X. 


\section{Oral exposure}

A sample size calculation was performed based on the formula described by Pandis. ${ }^{17}$ The parameters used for the calculation were the standard deviation by Silva et al., ${ }^{1} \alpha=0.05, \beta=0.2$, and a difference to be detected of $30 \%$ in coating loss percentage. This calculation indicated the need for 11 segments of each archwire and hence 11 individuals.

The selection criteria considered healthy patients with good oral hygiene, no caries, and complete permanent dentition. The ethical approval for this study was obtained from the ethics in research committee of Antônio Pedro University Hospital (process 37659714.3.0000.5243). Informed consent forms were signed by all patients.

All participants were already undergoing orthodontic treatment at Fluminense Federal University (UFF, Niterói, Brazil) and hold a metallic slot $0.022 \times 0.028$ " standard edgewise brackets (Morelli, Sorocaba, Brazil) in both arches.

Each subject received one 20-mm specimen of each archwire, which remained passively in place for 21 days in the oral cavity. The specimens were allocated randomly in the posterior region of both arches of each individual and tied juxtaposed to the pre-existing metal archwire, in passive engagement without any deflection, using gray elastic ligatures as figure eight elastic tie (Figure 2). Oral hygiene products (toothbrush and toothpaste) and instructions were given to all patients. The subjects were told not to use any other oral agents, such as mouthwashes, throughout the study.

All specimens were placed and removed by the same operator (N.A). Retrieved wires were collected after 21 days of oral exposure and individually immersed in detergent (Endozime AW Plus, Nova DFL, Rio de Janeiro, Brazil) in an ultrasound cleaner (Pro-Sonic 600,York, USA) for 30 minutes, so that organic debris could be removed. ${ }^{18}$ All the samples were submitted to the lab analyses.

\section{Coating loss measurement}

According to a methodology previously published, ${ }^{1}$ a graph paper was set on a glass slide to be used as a scale reference. All the 22 samples retrieved were fixed over the slide with wax on both ends. Two 5-mm-long steromicroscopy images were obtained for the same specimen, one on its right side and another on its left side, starting from the middle of the segment. Thus, the $10-\mathrm{mm}$ middle portion of each wire segment, corresponding to the interbracket space, was fully evaluated (Figure 3).

The overall area of the wire and the area corresponding to the coating loss on the labial surface were obtained using Image Pro Premier. Coating loss percentage was calculated from both measurements..

An individual adaptation for coating loss area measurements was required since depressions and convexities (shadows) on the surface of the coating were considered by the software as losses when the coverage was still in place. These automatic detections were removed by the operator.

\section{Scanning electron microscopy (SEM)}

A scanning electron microscope (Phenom ProX, Phenom World, Eindhoven, The Netherlands) was used to assess the micromorphological characteristics of the labial surface of three specimens of each type of wire (one as-received and two after oral exposure). The images were recorded at $15 \mathrm{kV}$, in backscattered mode using a charge reduction sample holder (low vacuum environment). The images were taken at 200X and 300X magnifications.

\section{D profilometry}

The topographic analysis of the coated surfaces of each archwire was performed using a 3D profilometer (Form Talysurf 60i, Taylor Hobson, Leicester, UK). An area of $0.24 \mathrm{~mm}^{2}(300 \times 800 \mu \mathrm{m})$ of each coated surface was scanned with a $20 \mathrm{~nm}$ z-resolution,

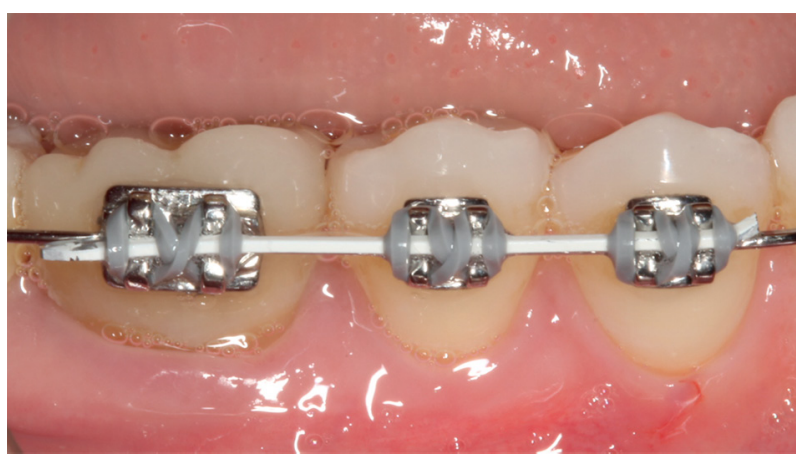

Figure 2. Esthetic coated wire in the oral environment. Wires were placed juxtaposed to the pre-existing metal archwire and tied with gray elastic ligatures as figure eight elastic tie. 
employing 40,000 steps in the $x$-axis and a spacing of $2 \mu \mathrm{m}$ in the $y$-axis. The reconstruction of 3 -D images was made according to the parameter $\mathrm{Sa}(\mu \mathrm{m})$ using the following formula:

$$
S a=\frac{1}{M N} \sum_{k=0}^{M-1} \sum_{I-0}^{N-1}\left|z\left(x_{k} ; y_{I}\right)\right|
$$

where $\mathrm{z}$ is the height of measured points in $\mathrm{x}$ and $\mathrm{y}$ coordinates.

\section{Statistical analyses}

All statistics were performed using BioEstat statistical software (version 5.0, Mamirauá Maintainable Development Institute, Belém, Brazil). A level of significance of 0.05 was adopted.

The same operator repeated coating thickness, wire dimensions and percentage of loss coating measurements with a one-week interval. The intraclass correlation coefficient (ICC) was used to confirm the calibration of the operator.

The Lilliefors test was used to confirm normality of the data. Coating thickness, wire dimensions and coating loss percentage were compared between the two wires using the independent $t$ test.

\section{Results}

The ICC (0.936) showed an excellent calibration of the researcher who performed the measurements.

Inner dimensions, coating thickness and the statistical differences for the as-received wires are shown in Table 1. The Biocosmetic wire presented, on an average, twice the coating thickness of the Titanol Cosmetic $(p<0.0001)$. Irrespective of this, both wires presented thinner coatings than those informed by the manufacturer $(0.002 ") .{ }^{14}$ On the
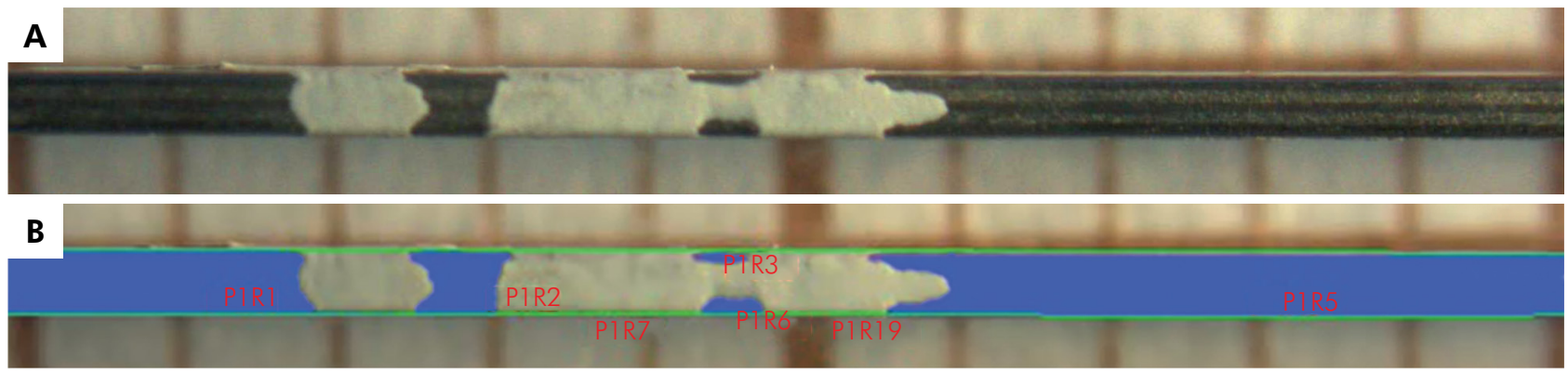

Figure 3. Stereomicroscope image of the labial surface of Titanol Cosmetic wire (10mm segment): A) before; and B) after the selection of coating loss area to be compared with the entire labial surface area. Original Magnification 14X.

Table 1. Wire dimensions, coating thickness, and statistical differences for as received wires.

\begin{tabular}{lccc}
\hline & Titanol Cosmetic & Biocosmetic & \\
\cline { 2 - 3 } Variable & $0.018 \times 0.024^{\prime \prime}$ & $0.018 \times 0.025^{\prime \prime}$ & $\begin{array}{c}\text { Difference } \\
\text { (p-value) }\end{array}$ \\
\hline Mean (SD) & Mean (SD) & $<0.0001^{*}$ \\
Inner height & $0.0162(0.0001)$ & $0.0140(0.0001)$ & $<0.0001^{*}$ \\
Coating thickness & $0.0229(0.0002)$ & $0.0211(0.0002)$ & $<0.0001^{*}$ \\
Total height & $0.0007(0.0002)$ & $0.0016(0.0003)$ & $p=0.0532$ \\
Total thickness & $0.0176(0.0002)$ & $0.0173(0.0004)$ & $p=0.0064^{*}$ \\
\hline
\end{tabular}

SD: std. deviation; *statistically significant difference. 
other hand, their total dimensions were comparable to those stated by the manufacturer.

The coating of the Biocosmetic wire was more uniform, including its edges, which differed from that of the Titanol Cosmetic, in which the lingual surface was not coated from the beginning and had a thinner coating on the corners (Figure 1).

Figure 4 shows the 3D profilometry images of the as-received wires. The Titanol Cosmetic sample (Figure $4 \mathrm{~A}$ ) presented a rougher surface with several peaks protruding from it. A more even, although irregular, surface with a striped pattern was observed in the Biocosmetic wire (Figure 4B).

The coating loss percentage after 21 days of oral cavity exposure for both tested wires is depicted in Table 2. The Titanol Cosmetic wire presented higher coating loss percentage than that of Biocosmetic. $(p=0.0346)$

Representative SEM images of the labial surface topography of as-received and retrieved wires are shown in Figure 5. The as-received Titanol Cosmetic wire (Figure 5A) revealed a surface totally coated but with a subtle and perceptible difference in shades of white color. Differently, the as-received Biocosmetic sample (Figure 5D) showed gray point-shaped areas. The retrieved segments of the two archwires showed different patterns of coating loss. The Titanol Cosmetic wire presented areas with gray appearance, suggesting partial abrasion of the coating (5B) and greater white areas with complete removal of the esthetic coating confirmed by a metallic exposure underneath (5C).
On the contrary, in both images of the Biocosmetic sample (5E and 5F), it is clear that although the Biocosmetic wire was partially or completely detached from the archwire surfaces, broader metallic areas could not be observed, and hence its esthetical attributes are less compromised.

\section{Discussion}

The Biocosmetic wire is a recent release that, according to its manufacturer, presents better esthetics and greater coating stability than its predecessor Titanol Cosmetic, which is still available for clinical use. This was the rationale to compare the performance of these two arches in the present study. In other words, if these improved characteristics of Biocosmetic wires are proved, its greater cost could be justified.

The lack of data is noticeable when studying the in vivo functionality of esthetic archwires. ${ }^{1,15}$ Clinical trials allow the analyses of retrieved wires and dental materials, where evidence is based on the environment in which it was intended to function. ${ }^{1}$

Table 2. Coating loss percentage after 21 days of oral cavity exposure.

\begin{tabular}{lccc}
\hline Variable & $\begin{array}{c}\text { Titanol } \\
\text { cosmetic }\end{array}$ & Biocosmetic & $\begin{array}{c}\text { Difference } \\
(p-\text { value })\end{array}$ \\
\hline Mean (SD) & $29.63(19.36)$ & $13.27(14.09)$ & \\
Cl & $17.97-41.29$ & $4.79-21.75$ & $0.0346^{*}$ \\
Minimum/maximum & $6-67$ & $0-44$ & \\
\hline
\end{tabular}

SD: std. deviation; $\mathrm{Cl}$ : Confidence Interval; * statistically significant difference.
A

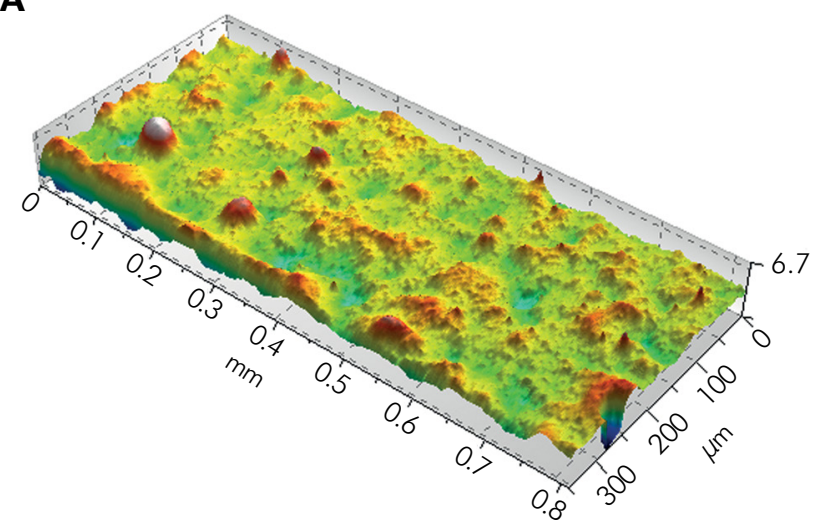

B

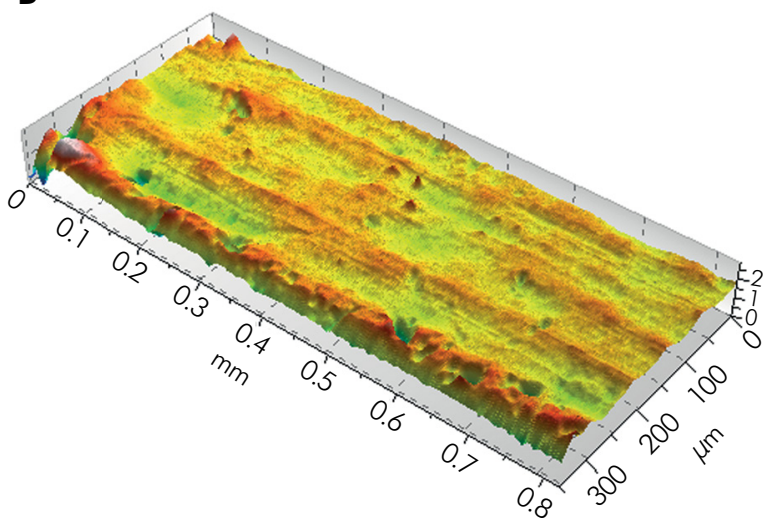

Figure 4. 3D profilometry images of A) Titanol Cosmetic; and B) Biocosmetic. 

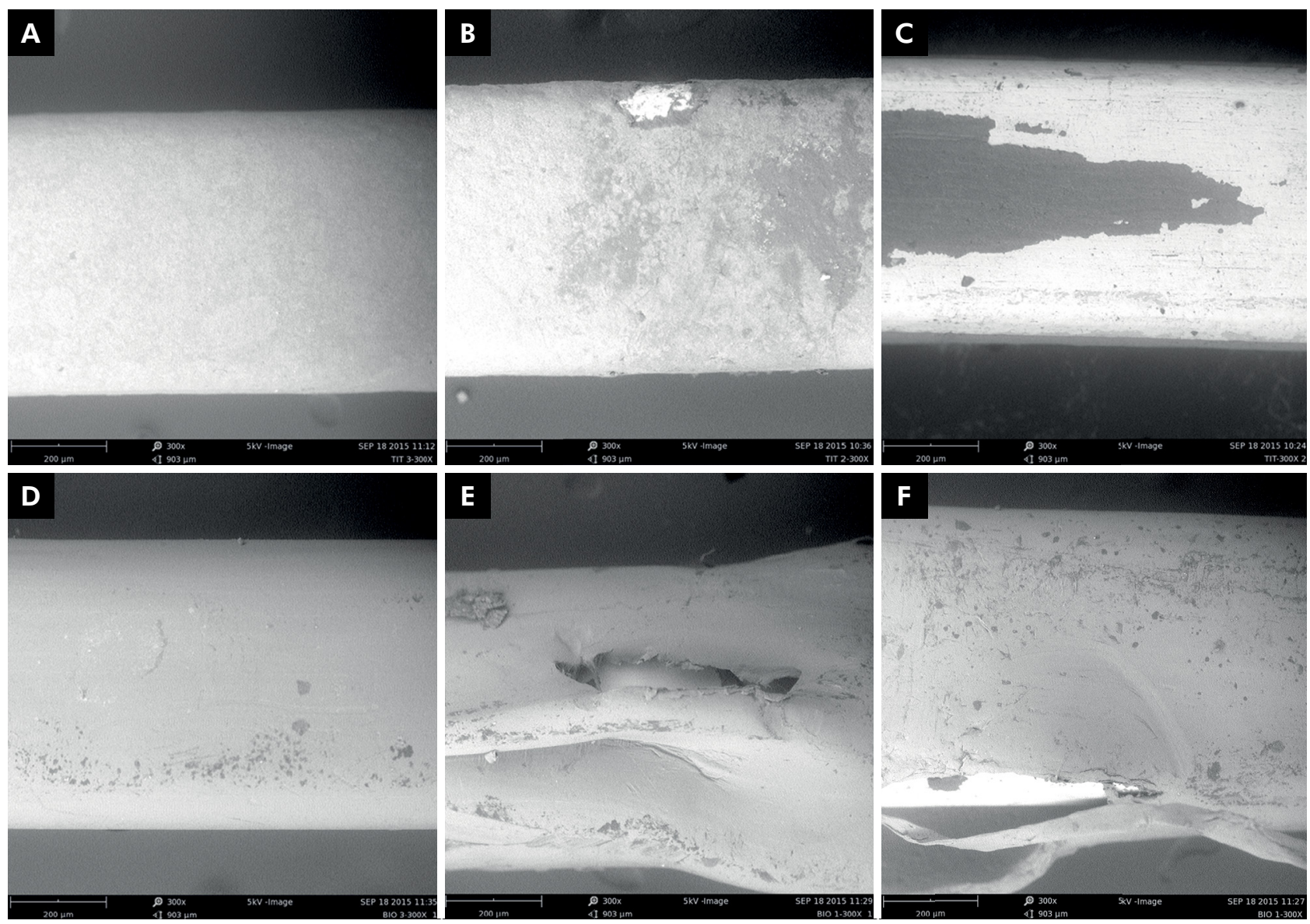

Figure 5. Photomicrographs of coated wires: A) as-received Titanol Cosmetic; B and C) postclinical Titanol Cosmetic; D) as-received Biocosmetic; E and F) postclinical Titanol Cosmetic. Original Magnification 300X.

Our results presented a statistically significant difference between Titanol Cosmetic and Biocosmetic samples when comparing inner (metallic) height, inner thickness, and coating thickness of the wires as-received from the manufacturer. This may be explained since the Biocosmetic wires presented approximately twice the coating thickness of Titanol Cosmetic ones. Nominal active dimensions can be affected by the increase of coating thickness. ${ }^{5,14,15}$ When coated and uncoated wires have the same overall dimensions, the cross-sectional area of the underlying metal of coated wires will be necessarily smaller. ${ }^{14}$ As described by Washington et al., ${ }^{14}$ Forestadent esthetic archwires are produced with a 0.002 " epoxy coating, a value that was not confirmed in our study (Table 1). Both wires tested in this study and the round 0.016 " cross-sectional wires from the same manufacturer tested previously ${ }^{14}$ showed smaller inner metallic dimensions than expected. In such cases, there may be less effectiveness and less force transfer values $^{14,19}$ which may influence treatment mechanics. ${ }^{15}$ However, when esthetic archwires are only coated on the labial surface, modification of the wire configuration is less probable and usual forces can be expected. ${ }^{19}$

Manufacturing specifications of esthetic wires are still scarce. ${ }^{1,14}$ Nevertheless, using SEM data related to material composition, visual characteristics and peeling results, we may infer that the esthetic coating application method is different in the two types of wires studied. This information complies with that reported by Rongo et al., ${ }^{4}$ who described that Titanol Cosmetic wire comprises a classical Teflon coating whereas the Biocosmetic is composed of a polymer coating.

An in vitro study, ${ }^{10}$ which compared uncoated traditional round cross-sectional (0.016") wires to equivalent esthetic coated wires, found best 
results for the Biocosmetic among the groups, in which the surface roughness was similar to the metallic wires but presented a higher friction. The Titanol Cosmetic group showed comparable friction to uncoated wires; however, its surface roughness was poorer. Although in the present study roughness of the two archwires was not evaluated, in our 3D profilometry images several peaks protruding from the Titanol Cosmetic surface could be noted in a scale from 0 to $6.7 \mu \mathrm{m}$ in the " $\mathrm{z}$ " axis (Figure 4A), reinforcing that this arch might be rougher than the Biocosmetic, which presented a scale of only 0 to $2 \mu \mathrm{m}$ (Figure $4 \mathrm{~B}$ ), matching the results from the aforementioned study. Friction between esthetic brackets that are commonly used in combination with esthetic archwires may be a key point in orthodontic treatment and, although not assessed in this study, should be considered. A previous study ${ }^{20}$ showed lower frictional forces in metal-insert ceramic brackets than in conventional ceramic brackets when being tested with metallic wires. Rudge et al. ${ }^{10}$ showed that most esthetic archwires presented a higher friction than that of the uncoated wires in metallic brackets. Sukh et al. ${ }^{21}$ reported that, when esthetic brackets and wires are considered, Teflon coated wires inserted in ceramic brackets with a metal slot show a lower friction than conventional ceramic brackets and even smaller than the combination between metallic wires and brackets.

In our study, coverage loss was identified in both types of wires. Considering the average orthodontic treatment duration, none of the archwires is ideal. However, the Biocosmetic wire was clinically more stable. Among the four types of archwires tested in a previous study with a similar methodology, ${ }^{15}$ Aesthetic Shiny Bright 0.018"x0.025" (TP Orthodontics) presented the best results with $28.71 \%$ of coating loss. This outcome was comparable with Titanol Cosmetic group in the current study (29.63\%), and whereas the Biocosmetic wire was even superior with only $13.27 \%$ of coating loss.

The better esthetic results for Biocosmetic wires compared to Titanol Cosmetic can be explained due to their conformation. Biocosmetic has a homogenous coverage on all surfaces whereas Titanol Cosmetic has no coating on the lingual surface and less coating on the edges. Clinically, it was clear that the peeling started exactly from the corners (Figure 3C), where tensile accumulation zones with lower coating thickness are probably located, and then it proceeded towards the center. This pattern is also in agreement with the findings of Silva et al. ${ }^{1}$ Areas with coating loss or deformation, whether with or without underneath metal exposure, showed surface defects which may contribute to plaque accumulation. ${ }^{1}$

As observed after 21 days of oral exposure, the polymer coating of the Biocosmetic wire was generally still in place, even when partly detached from the wire, as it was connected to the remaining coating. When observing the retrieved specimens through a stereomicroscope, the metal and the coating were no longer adhered, but the underlying metal was not visible. According to this feature, when calculating the coating loss percentage, areas as described above were considered esthetic, as from a clinical point of view the coverage was still in place. Nevertheless, this detachment may prejudice sliding mechanics and should be tested in future studies.

The acquired results indicate enhancement in orthodontic rectangular esthetic archwires, since the Biocosmetic group proved to be more stable than other wires already tested. ${ }^{1}$ However, none of the studied wires have ideal esthetic features as most of the samples did not remain intact after 21 days, an extremely short period of time when being compared to the period in which a rectangular archwire may be maintained. ${ }^{1}$

It could be questionable whether the use of an ultrasonic bath to remove organic debris from the wires previously to their assessment could lead to further coverage loss. The specific method used was based on a previous study also on esthetic coated orthodontic archwires. ${ }^{1}$ In addition, similar methods are reported in the literature related to the cleaning of stainless steel orthodontic wires ${ }^{22}$ and photographic retractors. ${ }^{23}$

In this study, the fact that only segments were used instead of the entire archwire may have made the clinical simulation incomplete, representing a limitation. Moreover, the segments were not inserted in the bracket slot; therefore, no wire deflection was 
induced, as would be observed in cases of leveling and aligning. Since esthetic brackets were not used, future studies should test esthetic wires combined with ceramic or plastic brackets, which would increase the clinical application of the results. More clinical trials are then necessary to enhance literature when it comes to esthetic archwires that are already commercially available and to improve these materials until ideal esthetic aspects are developed.

\section{References}

1. Silva DL, Mattos CT, Simão RA, Ruellas ACO. Coating stability and surface characteristics of esthetic orthodontic coated archwires. Angle Orthod. 2013;83(6):994-1001. https://doi.org/10.2319/111112-866.1

2. Elayyan F, Silikas N, Bearn D. Ex vivo surface and mechanical properties of coated orthodontic archwires. Eur J Orthod. 2008;30(6):661-7. https://doi.org/10.1093/ejo/cjn057

3. Russell JS. Aesthetic orthodontic brackets. J Orthod. 2005;32(2):146-63. https://doi.org/10.1179/146531205225021024

4. Rongo R, Valletta R, Bucci R, Rivieccio V, Galeotti A, Michelotti $A$ et al. In vitro biocompatibility of nickel-titanium esthetic orthodontic archwires. Angle Orthodontist. 2016;86(5):789-95. https://doi.org/10.2319/100415-663.1

5. Elayyan F, Silikas N, Bearn D. Mechanical properties of coated superelastic archwires in conventional and self-ligating orthodontic brackets. Am J Orthod Dentofacial Orthop. 2010;137(2):213-7. https://doi.org/10.1016/i.ajodo.2008.01.026

6. Lopes Filho H, Maia LE, Araújo MV, Ruellas AC. Influence of optical properties of esthetic brackets (color, translucence, and fluorescence) on visual perception. Am J Orthod Dentofacial Orthop. 2012;141(4):460-7. https://doi.org/10.1016/i.ajodo.2011.10.026

7. Wichelhaus A, Geserick M, Hibst R, Sander FG. The effect of surface treatment and clinical use on friction in NiTi orthodontic wires. Dent Mater. 2005;21(10):938-45. https://doi.org/10.1016/i.dental.2004.11.011

8. Husmann P, Bourauel C, Wessinger $M$, Jäger $A$. The frictional behavior of coated guiding archwires. J Orofac Orthop. 2002;63(3):199-211. https://doi.org/10.1007/s00056-002-0009-5

9. Doshi UH, Bhad-Patil WA. Static frictional force and surface roughness of various bracket and wire combinations. Am J Orthod Dentofacial Orthop. 2011;139(1):74-9. https://doi.org/10.1016/j.ajodo.2009.02.031

10. Rudge P, Sherriff M, Bister D. A comparison of roughness parameters and friction coefficients of

\section{Conclusions}

The Biocosmetic coating was thicker and more uniform, whereas the Titanol Cosmetic coating had no coverage on the lingual surface and with a thinner coating on the edges.

After oral exposure, both tested wires presented a significant coating loss but the Biocosmetic wire revealed better esthetic results.

aesthetic archwires. Eur J Orthod. 2015;37(1):49-55.

https://doi.org/10.1093/ejo/cju004

11. Postlethwaite KM. Advances in fixed appliance design and use:

1. Brackets and archwires. Dent Update. 1992;19(7):276-80.

12. Kusy RP. A review of contemporary archwires: their properties and characteristics. Angle Orthod. 1997;67(3):197-207.

13. Neumann P, Bourauel C, Jäger A. Corrosion and permanent fracture resistance of coated and conventional orthodontic wires. J Mater Sci Mater Med. 2002;13(2):141-7. https://doi.org/10.1023/A:1013831011241

14. Washington B, Evans CA, Viana G, Bedran-Russo A, Megremis S. Contemporary esthetic nickel-titanium wires: do they deliver the same forces? Angle Orthod. 2015;85(1):95-101. https://doi.org/10.2319/092513-701.1

15. Silva DL, Mattos CT, Sant' Anna EF, Ruellas AC, Elias CN. Cross-section dimensions and mechanical properties of esthetic orthodontic coated archwires. Am J Orthod Dentofacial Orthop. 2013;143(4 Suppl):S85-91. https://doi.org/10.1016/j.ajodo.2012.09.009

16. Silva DL, Mattos CT, Araújo MV, Ruellas ACO. Color stability and fluorescence of different orthodontic esthetic archwires. Angle Orthod. 2013;83(1):127-32. https://doi.org/10.2319/121311-764.1

17. Pandis N, Polychronopoulou A, Eliades T. Sample size estimation: an overview with applications to orthodontic clinical trial designs. Am J Orthod Dentofacial Orthop. 2011;140(4):e141-6. https://doi.org/10.1016/i.ajodo.2011.04.021

18. Izquierdo PP, Biasi RS, Elias CN, Nojima LI. Martensitic transformation of austenitic stainless steel orthodontic wires during intraoral exposure. Am J Orthod Dentofacial Orthop. 2010;138(6):714.el-5. https://doi.org/10.1016/j.ajodo.2010.05.015

19. Kaphoor AA, Sundareswaran S. Aesthetic nickel titanium wires: how much do they deliver? Eur J Orthod. 2012;34(5):603-9. https://doi.org/10.1093/ejo/cjr089 
20. Cacciafesta V, Sfondrini MF, Scribante A, Klersy C, Auricchio F. Evaluation of friction of conventional and metal-insert ceramic brackets in various bracket-archwire combinations. Am J Orthod Dentofacial Orthop. 2003;124(4):403-9. https://doi.org/10.1016/S0889-5406(03)00501-8

21. Sukh R, Singh GK, Tandon P, Singh GP, Singh A. A comparative study of frictional resistance during simulated canine retraction on typodont model. J Orthod Sci. 2013;2(2):61-6. https://doi.org/10.4103/2278-0203.115091
22. Normando D, Araúio AM, Marques IS, Dias CGBT, Miguel JA. Archwire cleaning after intraoral ageing: the effects on debris, roughness, and friction.

Eur J Orthod 2013;35(2):223-9. https://doi.org/10.1093/ejo/cjr104

23. Benson PE, Ebhohimen A, Douglas I. The cleaning of photographic retractors; a survey, clinical and laboratory study. Br Dent J 2010;208(7):E14. https://doi.org/10.1038/sj.bdj.2010.310 